\title{
Biliary Intraepithelial Neoplasia-2
}

National Cancer Institute

\section{Source}

National Cancer Institute. Biliary Intraepithelial Neoplasia-2. NCI Thesaurus. Code C67492.

Biliary intraepithelial neoplasia characterized by the presence of moderate epithelial atypia. 